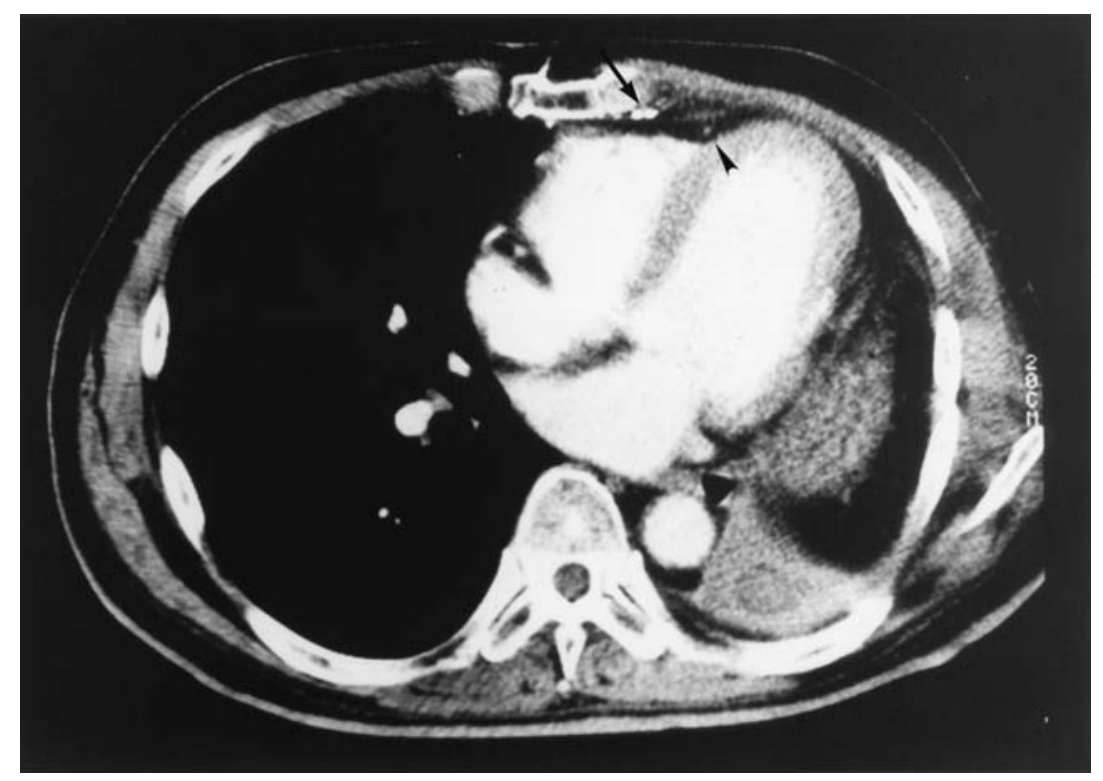

Fig. 1. Enhanced ultrafast $\mathrm{CT}$ scan of a patient with a previous sternotomy for CABG and left thoracotomy for pneumonectomy. The LAD (arrowhead) is superficial and shifted medially and anteriorly near the left ITA (arrow).

depth of the LAD can be assessed and intramuscular arteries can be excluded from the MICABG approach before the operation. Furthermore, a minimum-sized thoracotomy can be made just above the LAD, allowing minimal dissection of pleural or pericardial adhesions in patients who have had previous sternotomies or thoracotomies.

\section{REFERENCES}

1. Boonstra PW, Grandjean JG, Mariani MA. Improved method for direct coronary grafting without $\mathrm{CPB}$ via anterolateral small thoracotomy. Ann Thorac Surg 1997;63:567-9.
2. Acuff TE, Landreneau RJ, Griffith BP, Mack MJ. Minimally invasive coronary artery bypass grafting. Ann Thorac Surg 1996;61:135-7.

3. Landreneau RJ, Mack MJ, Magovern JA, et al. "Keyhole" coronary artery bypass surgery. Ann Surg 1996;224:453-62.

4. Ohtsuka T, Wolf RK, Hiratzka LH, Wurnig P, Flege JB Jr. Thoracoscopic internal mammary artery harvest for MICABG using the Harmonic Scalpel. Ann Thorac Surg 1997;63:S107-9.

\title{
SYNDROME X: RELIEF BY TRANSMYOCARDIAL REVASCULARIZATION
}

Allan M. Lansing, MD, PhD, Louisville, Ky.

From The Heart Institute, Audubon Regional Medical Center, Louisville, Ky.

Received for publication Feb. 10, 1998; accepted for publication March 3, 1998.

Address for reprints: Allan M. Lansing, MD, PhD, The Heart Institute, Audubon Regional Medical Center, One Audubon Plaza Dr., Louisville, KY 40217.

J Thorac Cardiovasc Surg 1998;116:174-6
Syndrome X, a term used by Kemp, ${ }^{1}$ and the more descriptive term "microvascular angina" 2 are used to describe cases of typical angina, normal coronary arteries, and no other cardiac cause for angina such as mitral valve prolapse, ventricular hypertrophy, aortic valve disease,

Copyright $(0) 1998$ by Mosby, Inc.

$0022-5223 / 98 \$ 5.00+0 \quad \mathbf{1 2 / 5 4 / 9 0 0 1 0}$ 
pulmonary hypertension, or myocardial bridging. The chest pain is frequently prolonged and poorly relieved by nitroglycerin. A patient with this syndrome was treated by transmyocardial revascularization (TMR) with the carbon dioxide laser years ago with complete relief of symptoms.

Patient and methods. A 54-year old female registered nurse was referred with chest pain that had occurred at rest and with exertion since early 1992 . She had a history of hypertension, hyperlipidemia, irritable bowel syndrome, and hysterectomy. She had a strong family history of cardiac disease, her father having had a myocardial infarction and having died of a stroke. She had smoked one pack of cigarettes per day for 30 years. Cardiac catheterization on March 10, 1994, revealed entirely normal coronary arteries with no focal areas of stenosis but a small-caliber left anterior descending coronary artery. A nuclear study on April 9, 1992, showed reversible ischemia anteriorly. She had multiple admissions to the hospital in Virginia with prolonged chest pain associated with sweating and ST segment depression, which were managed with intravenous heparin and nitroglycerin. She was on a regimen of fluoxetine hydrochloride (Prozac) $20 \mathrm{mg}$, conjugated estrogen (Premarin) $0.625 \mathrm{mg}$, verapamil (Calan SR) $180 \mathrm{mg}$, amlodipine besylate (Norvasc) $10 \mathrm{mg}$, atenolol (Tenormin) $25 \mathrm{mg}$, sublingual nitroglycerin, and the combination drug chlordiazepoxide hydrochloride and clidinium bromide (Librax) for irritable bowel syndrome. Results of her physical examination were within normal limits, with all peripheral pulses being present, no bruits, normal cardiac findings, a blood pressure of $130 / 80 \mathrm{~mm}$ $\mathrm{Hg}$, and sinus rhythm. The ejection fraction was $75 \%$ by echocardiogram, and at the time of transfer she was receiving intravenous heparin and nitroglycerin.

The woman was not a candidate for angioplasty or coronary bypass grafting, and multiple medications used over a 2-year period had been unsuccessful in controlling symptoms. Consequently, she was accepted for TMR and was taken to the operating room on August 5, 1994, while still receiving intravenous medication. The entire left ventricle was treated, but because of significant fat over the apex of the heart, only 26 of 34 laser channels successfully reached the left ventricle as confirmed by the transesophageal echocardiogram (TEE). The TEE confirmed excellent left ventricular function and normal mitral valve function during the operative procedure. She had been receiving aspirin therapy with a prolonged bleeding time before the operation and was given 5 units of platelets at the end of the procedure.

In the immediate postoperative period she had pulmonary congestion and finally pulmonary edema and could not be weaned from the ventilator. A systolic murmur appeared at the apex and an echocardiogram on August 8 showed good left ventricular function but significant mitral regurgitation. A TEE on August 9 showed gross mitral regurgitation and suggested ruptured chordae tendineae. Consequently, she was taken back to the operating room on August 10,1994, at which time the mitral valve was repaired. A portion of the posterior leaflet was resected and a No. 31 Duran ring annuloplasty was done. Two laser holes were noted in the left ventricle at the site where the chordae tendineae had been injured during the operation and subsequently ruptured the next day. A postoperative echocardiogram showed good ventricular function and no mitral regurgitation, and she was discharged on August 21.

Follow-up at 3 months and at 6 months indicated that she had class III angina with normal left ventricular function and only a trace of mitral regurgitation. By 1 year she had no angina, the ejection fraction remained $65 \%$ to $70 \%$ with the trace of mitral regurgitation that had been present before the operation, and the thallium scan was reported as within normal limits. She has had no recurrence of angina or unstable angina in the subsequent years and has not been readmitted to the hospital, whereas she had required monthly visits to the emergency department before the operation.

Discussion. The mechanism of syndrome $X$ has been extensively studied but remains a mystery. Cannon and associates ${ }^{3}$ believe that an abnormal flow reserve resulting from changes in the microcirculation is responsible. These vessels are too small to be visualized by coronary angiography, and no definitive changes have been defined by endomyocardial biopsy. Thallium studies show abnormalities in only a few patients, probably because the ischemia is diffuse and not localized, so that isotopic detection is difficult. Precipitation of chest pain by atrial pacing and intravenous ergonovine have been tried with suggestive but not conclusive results to indicate less coronary flow and less coronary dilation during these stimuli. ${ }^{3}$ Patients with syndrome $\mathrm{X}$ have normal left ventricular function on exertion despite the occurrence of angina and ST segment depression. These studies suggest that angina may develop without myocardial ischemia in patients with syndrome $\mathrm{X}$ as a result of abnormal cardiac sensitivity. ${ }^{4}$ Inasmuch as inadequate vasodilator capacity of the microcirculation is suspected to be the cause, calcium channel blockers, nitrates, and $\beta$-blockers have been used in therapy.

TMR was tried in this patient because all medical measures had failed and the patient was incapacitated. In the past 4 years we have used TMR for the relief of class IV angina unresponsive to medical therapy and unsuitable for coronary bypass or angioplasty in 295 patients. We used a carbon dioxide laser in 228 and a holmiumyttrium-aluminum-garnet laser in 67 . The results of these studies were documented in a recent report. ${ }^{5}$ Our observations indicate that denervation and the reptilian theory play at most a minor part in the relief of angina, as opposed to vascular neogenesis and connection to intramyocardial collaterals. In addition, different lasers have different effects. However, inasmuch as we are not sure about the cause of syndrome $\mathrm{X}$, it is difficult to propose a mechanism to explain the dramatic result obtained in this case.

Finally, this patient was the unfortunate victim of a rare complication of TMR. Two of the chordae supporting the posterior mitral valve leaflet were injured, presumably because they were lying immediately adjacent to the left ventricular endocardium and hence were not protected from the laser beam by blood in the left ventricle. Perforation of a mitral valve leaflet is occasionally seen, but the $1 \mathrm{~mm}$ hole rarely causes any significant complication. A ruptured chorda has been described on occasion, but so far as we know this is the only patient who has 
required mitral valve repair. Fortunately, the repair was entirely successful and the patient remains free of symptoms from the standpoint of both angina and mitral regurgitation.

\section{REFERENCES}

1. Kemp HG. Left ventricular function in patients with the anginal syndrome and normal coronary arteriograms. Am J Cardiol 1973;32:375-6.

2. Arbogast R, Bourassa MG. Myocardial function during atrial pacing in patients with angina pectoris and normal coronary arteriograms: comparison with patients having significant coronary artery disease. Am J Cardiol 1973;32:257-63.

3. Cannon RO, Watson RM, Rosing DR, Esptein SE. Angina caused by reduced vasodilator reserve of the small coronary arteries. J Am Coll Cardiol 1983;6:1359-73.

4. Cannon RO, Quyyumi AA, Schenke WH, Fananapazir L, Tucker EE, Gaughan AM, et al. Abnormal cardiac sensitivity in patients with chest pain and normal coronary arteries. J Am Coll Cardiol 1990;16:1359-66.

5. Lansing AM. Transmyocardial revascularization: mechanism of action with carbon dioxide and holmium-yttrium-aluminum-garnet lasers [letter]. J Thorac Cardiovasc Surg 1998;115: 1392.

\title{
ENDOGENOUS ENDOPHTHALMITIS RESULTING FROM STERNAL WOUND INFECTION AFTER CORONARY ARTERY BYPASS GRAFTING
}

\author{
Suresh K. Nahata, MD, Norman A. Saffra, MD, Mark H. Genovesi, MD, Mark W. Connolly, MD, and \\ Joseph N. Cunningham, Jr., MD, Brooklyn, N.Y.
}

Endogenous endophthalmitis is an uncommon complication after surgery, but its incidence is increasing because of increasing use of immunosuppressive drugs, spread of acquired immunodeficiency syndrome, and increased use of invasive procedures. The purpose of this case report is to alert cardiac surgeons about this complication, because a patient's vision can be saved only with an aggressive early intervention by an ophthalmologist. ${ }^{1}$

Case report. A 56-year-old man with non-insulin-dependent diabetes mellitus underwent uneventful double coronary artery bypass grafting in June 1996. A week after discharge from the hospital, the patient was readmitted with a sternal wound infection. He was empirically started on intravenous (IV) vancomycin and aztreonam (Azactam). Gram stain showed gram-positive cocci and culture grew Staphylococcus aureus. The patient was brought to the operating room and had wound debridement, internal fixation of the sternum, and pectoralis minor flaps for wound closure.

On the third postoperative day he started having pain in the right eye. The white blood cell count rose from 8,000 to $13,000 / \mathrm{mm}^{3}$ and a spiking fever developed. An urgent ophthalmology consultation was requested. On examina-

From the Department of Surgery, Maimonides Medical Center, 4802 Tenth Ave., Brooklyn, N.Y.

Received for publication Feb. 23, 1998; accepted for publication Feb. 27, 1998.

Address for reprints: Norman A. Saffra, MD, 921 49th St., Brooklyn, NY 11219.

J Thorac Cardiovasc Surg 1998;116:176-7

Copyright (c) 1998 by Mosby, Inc.

0022-5223/98 \$5.00+ $0 \quad \mathbf{1 2 / 5 4 / 9 0 1 9 4}$ tion, the right eye had hypopyon with a hazy vitreous and fundus and no red reflex. The vision was 20/70 in the right eye. The left eye had no abnormalities. A diagnosis of endogenous endophthalmitis was made and the patient was taken to the operating room on an emergency basis. The patient had an anterior chamber tap and pars plana vitrectomy with intravitreal injection of $1 \mathrm{mg}(0.1 \mathrm{ml})$ of vancomycin and $400 \mu \mathrm{g}(0.1 \mathrm{ml})$ of dexamethasone. He also received subconjunctival injections of vancomycin and dexamethasone, and both the anterior chamber and vitreous were cultured. In the postoperative period, the patient continued to receive IV vancomycin, and he was also started on a program of vancomycin, prednisolone, and cyclopentolate eye drops and the combination drug dexamethasone, neomycin, and polymyxin B (Maxitrol) eye ointment.

The patient continued to have spiking fever and the white blood cell count did not decrease. The sternotomy wound showed increased erythema, and we decided to return him to the operating room. Four days after the eye operation he had further debridement of the sternotomy wound with removal of screws and plates and with bilateral pectoralis major muscle flaps for wound closure. The cultures from the vitreous and anterior chamber showed no growth. At this time the patient's visual acuity and general condition continued to improve. The visual acuity $2 \frac{1}{2}$ weeks after the eye operation was $20 / 30$ in the right eye. The patient was discharged to his home 3 weeks after his admission with instructions to continue IV antibiotics for 6 more weeks.

Discussion. Endophthalmitis is an intraocular infection that can occur exogenously, as after ocular surgery or trauma (organisms introduced from outside), or endogenously (the primary focus of infection being within the 